\section{Aging changes complexity of heart rate dynamics assessed by entropy and Lyapunov exponent analysis}

\author{
Roberto Siciliano, ${ }^{1}$ Giuseppe Agapito, ${ }^{2}$ \\ Sarah Siciliano, ${ }^{3}$ \\ Filippo Luca Fimognari ${ }^{4}$ \\ ${ }^{1}$ Medical Physics Unit, Cosenza \\ Hospital, and Applied Physics, Magna \\ Graecia University, Catanzaro; \\ ${ }^{2}$ Department of Medical and Surgical \\ Science, Magna Graecia University, \\ Catanzaro; ${ }^{3}$ Physicist, Cosenza; \\ ${ }^{4}$ Geriatrics Unit, Department of Internal \\ Medicine, Cosenza Hospital, Italy
}

\section{Abstract}

In recent years, many research groups are trying to quantify the physiological signals of an individual, proposing new models to assess the complex dynamics of biological control systems. Indeed, life coincides with the good handling of the structures in the organism and of physiological control mechanisms, while disease and death coincide with the loss of structure and of coordinated functions. The homeodynamic systems which normally govern health are the same that cause pathological events when activated inadequately, or rather, when the balance between order and chaos of the elementary physiological processes is no longer effectively controlled in relation to any type of stress, both external and internal to the body. In a complex system, loss or alteration of communication between physiological signals means pathology.

In this paper a signal analysis method based on Entropy (E), Lyapunov exponent (1), Median Absolute Deviation (MAD), Multiscale Entropy (MSE), is proposed to estimate the complexity of long-range temporal correlation heart rate (HR) time series for an elderly person and a young person both healthy. These new methods could improve overall understanding of the physiological system of the human organism, by adopting new models and experimental paradigms, such as those of fractality and entropy, who have the ability to direct from an organ medicine to a modern systemic medicine.

\section{Introduction}

Currently the concept of aging can be described as a less individual capacity to deal with new environmental stress situations.

This reduced ability of continuous adaptations manifests it self through a progressive deterioration of the control mechanisms which supervise the physiological functions that take place and interact at the sub-cellular, cellular, histological and organ levels, is the basic mechanism to going towards a condition of less health.

Therefore, the aging of an individual is usually a continuous collection of health problems that increase with a personal frequency.

The concept of frailty, in recent years, has found its place in the international community to describe the risk of compromised health status of an individual. ${ }^{1-2}$ Frailty is not intended in itself as a disease state, but an increase in the risk of contract an illness, associated with alterations of the complex network of human physiological functions.

In this conceptual framework, frailty is not a disease sinsu stricto, but an intermediate position between a good functional state and a deteriorated state, or between a state of health and one of disease.

Other authors $\mathrm{s}^{3-5}$ have imagined the individual fragility as a number of deficits accumulated in the course of its existence.

In past years many scientists have believed that living systems, as also other fields of study, could be understood with a reductionist approach. Indeed, reductionism has obtained huge successes in recent decades in many other scientific disciplines, in particular, the idea was to analyze small parts of the entire biological systems, considered entirely too complex. But, despite the in-depth knowledge of subsets of the system under study, the full understanding of the biological system has not reached, and it is now clear that we must have an integrated point of view, a model to explain the whole phenomenon.

Other authors ${ }^{6,7}$ have believed that a more precise approximation of real state of health or aging of an individual is possible if the level of order or complexity of the anatomical apparatus is known through the evaluation of entropy.

\section{Data analysis}

Software pipelines are becoming essential in data analysis especially in analyzing wave e.g, electroencephalogram (EEG), electrocorticogram (ECoG), electrocardiogram (ECG), electromyogram (EMG), etc. The wave analysis provides to recognize repetitive patterns that could be used to learn better knowledge concerning the problem under investigation. In medical areas, waves analysis is a very popular task especially in neurology, and cardiology
Correspondence: Roberto Siciliano, Medical Physics Unit, Cosenza Hospital, and Applied Physics, Magna Graecia University,

Catanzaro, Italy.

E-mail: robsic65@gmail.com

Key words: Aging; fractals; entropy; Lyapunov exponent; nonlinear dynamics.

Received for publication: 17 January 2019. Accepted for publication: 29 March 2019.

This work is licensed under a Creative Commons Attribution-NonCommercial 4.0 International License (CC BY-NC 4.0).

CCopyright R. Siciliano et al., 2019

Licensee PAGEPress, Italy

Geriatric Care 2019; 5:8038

doi:10.4081/gc.2019.8038

where most of the clinical-report are obtained after analyzing ECG and ECC. Thus, in these areas automatic wave analysis tools are fundamental.

As a general practice, the researchers are supported only in the first phases of waves management through simple tools for memorization and visualization of the raw data. On the other hand, there is a lack in software tools can perform waves analysis automatically. Indeed studies have to be completed manually especially in studies where it is necessary to compare several datasets. Multiple data comparison is a long and challenging process and requires very expert figure to attribute a meaning to the waves, as well as an error-prone process. The complexity is evident in the analysis of a single ECG raw data to figure out recurrent patterns in the signals, that after can be used to detect heart failure, arrhythmias, and so on. The evaluation becomes even more complicated if the researcher needs to compare a population of ECGs to make it possible to use the pattern hidden in the ECG diagrams to discriminate features related with particular classes (i.e., healthy or diseased) of the population.

The general process of signals acquisition is depicted in Figure 1.

There are several software tools with which to perform signal analysis, ${ }^{8-10}$ each tool is designed to perform the analysis from signal obtained through the electrodes values.

These software have been designed for physiologic signal processing and analysis and detection of physiologically significant events. Some of these have been implemented using Matlab environment.

All these software can perform analysis on the signal but lacking in tool to compute entropy or other complex physical measures. 
To compute physical quantities on ECG data, the researchers have to use multiple software tools taking care to make the output of the previous tool in a compatible format for the next one. The creation of software programs can be a long and difficult task especially for users with low IT skills.

To make faster to analyze ECG data we developed and implemented a library of software programs in the Python 3.6 environment to analyze multiple raw data signals. The analysis process is organized as a pipeline where the output of the first module becomes the input for the next one.

The files are read one at a time and analyzed through functions that attempts to locate QRS complexes in an ECG signal. The results are automatically given as input to the subsequent functions, that allow to calculates the instantaneous heart rate. Finally, the results (time series) are saved on the file system into the specified directory.

The second module of the implemented pipeline, reading the data saved from the previous module, convert the signal data into an heart rate chart saving each single chart in the file system. Thus, the different charts can be analyzed and compared.

The final module of the pipeline is the Analyser module that for each single time series compute the entropy, lyapunov exponent, median absolute deviation, and multiscale entropy values to highlight repetitive patterns into the ECG that can be used to discriminate among subjects belong to different classes.

It is worthy to note that the whole implement signal data analysis workflow is done automatically.

They must only be provided the directory of the file system where the data to analyze are stored, e the output directory where results must be saved.

\section{Materials and Methods}

To contribute to the understanding of the complex dynamics in the biophysical signals we perform analyses of heart rate variability (HRV) signals through the Entropy, Lyapunov exponent, Median Absolute Deviation and Multiscale Entropy parameters.

Entropy has been a common index to quantify the complexity of time series in a variety of fields. Here, we consider entropy to measure the complexity and disorder degree of time series. Simulations on synthetic data and tests on epileptic EEG signals have demonstrated its ability of detecting the abrupt change, regardless of energetic (e.g. spikes or bursts) or structural changes.

In recent years, entropy has been widely applied to quantify the complexity of a variety of experimental time series and in particular physiological signal.

We focus on the variations of $\mathrm{E}$ as the magnitude of entropy indicate of dynamic changes hidden in the signal.

Lyapunov exponents 1 which provide a qualitative and quantitative characterization of dynamical behavior, are related to the exponentially fast divergence or convergence of nearby orbits in phase space. A system with one or more positive Lyapunov exponents is defined to be chaotic.

Here we consider the Lyapunov exponents, which has proven to be the most useful dynamical diagnostic for chaotic systems. Lyapunov exponents are the average exponential rates of divergence or convergence of nearby orbits in phase space.

Since nearby orbits correspond to nearly identical states, differently, exponential orbital divergence means that systems

with initial differences we may not be able to resolve will soon behave quite differently and predictive ability is rapidly lost. Any system containing at least one positive Lyapunov exponent is defined to be chaotic.

In mathematics, the Lyapunov time $\left(\mathrm{T}_{1}\right)$ is the characteristic timescale on which a dynamical system becomes chaotic. $T_{1}$ is defined as the inverse of a system's largest Lyapunov exponent. The Lyapunov time mirrors the limits of the predictability of the system.

The method of multiscale entropy (MSE) analysis is useful for investigating complexity in physiologic signals and other series that have correlations at multiple (time) scales. ${ }^{11,12}$ MSE analysis is a method of measuring the complexity of time series. This tool can be applied both to physical and physiologic time series. MSE can be used with differents method for calculating the entropy. We have used MSE, where entropy is evaluated using the sample entropy (SampEn). ${ }^{13}$ SampEn is a refinement of the approximate entropy family introduced by Pincus. ${ }^{14}$ Both have been widely used for the analysis of physiologic data sets. ${ }^{15,16}$ A high MSE value indicates a more complex time series.

The median absolute deviation is a measure of statistical dispersion. The MAD is a robust measure of the variability of a sample of quantitative data. Moreover, the MAD is a robust statistic, being more resilient to outliers in a data set than the standard deviation. In the standard deviation, the distances from the mean are squared, so large deviations are weighted more heavily, and thus outliers can heavily influence it. In the MAD, the deviations of a small number of outliers are irrelevant.

\section{Results and Discussion}

An important question is whether the heterogeneous physiologic time series arises from external or intrinsic stimuli, which carry the system away from a homeostatic state. An suggestive alternative is ipothesis that physiologic fluctuations are, at least in part, due to the underlying dynamics of the system.

The aging process causes a continuous deterioration of control systems of the human body, which are present at different levels: molecular, cellular, organ and systemic. These control mechanisms govern the complex network of physiological functions.

The processes is continuous, and in this way the body responds immediately to any type of environmental stress through a process called oleodynamic, ${ }^{17}$ introduced by Yates, ${ }^{18}$ which aims to bring back the system to the normal state of homeostasis through the continuous interaction of several mechanisms of regulation and control; ${ }^{19,20}$

Recently, several authors have proposed new concepts to describe the dynamics of physiological systems in order to predict future health states. ${ }^{21-23}$ These concepts, are generally based on the use of methods used

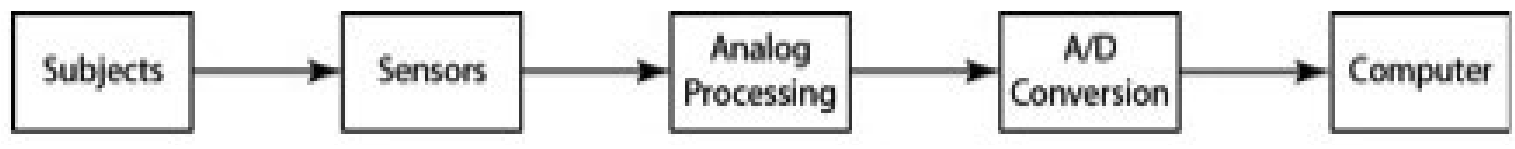

Figure 1. The general waves acquisition process. 
in physics in the field of nonlinear dynamics (chaos theory) and statistical physics and the use of concept of fractals ${ }^{24}$ to try to understand, quantify and model the aging of human body, ${ }^{25,26}$ seen as a variation of the complexity of physiological dynamics. In fact, it has been observed that different physiological processes (heart rate, anatomy of the respiratory tract, vascular system and the intricate network of the neurological system) have fractal properties (temporal and spatial) of self-similarity; ;7,28 $^{27}$ therefore, it is becoming clear that it is necessary to consider them as complex systems, and their development over time, cannot be fully understood without using new techniques.

A proper method to understand the complexity of a system is to measure entropy. This method is conceptually linked to the classical physics of a thermodynamic system and indicates the degree of overall order of the molecules that compose it.

Complex systems can give rise to collective behaviours, which are not simply the sum of their individual components ${ }^{29}$ but involve any single units constantly interacting with their environment. The way in which this happens is still a mystery. Understanding the emergence of ordered behaviour of spatio-temporal patterns and adaptive functions appears to require global concepts and tools.

Beyond its common use, the physical meaning of the word chaos does not mean disorder or confusion. It indicates a specific system with dynamical behaviour. Today, several methods are available to character- ize chaotic systems. An important physical quantity is just entropy. ${ }^{30}$

Entropy is a major thermodynamic quantity describing the amount of order in a system, and it supplies an important approach for the analysis of a system that evolves in the time, which can be regarded as a source of information. From a microscopic point of view, the second law of thermodynamics tells us that a system tends to evolve towards a condition, which has the largest number of accessible states compatible with the macroscopic conditions. Maximum entropy corresponds to the maximum number of possible microscopic positions (high fractal complexity). ${ }^{31}$

In recent years, many researchers and clinicians investigating human aging noticed that a loss of fractal complexity of anatomical structures and physiological processes, have used the concept of entropy to estimate changes in fractal complexity or irregularities in the dynamics of physiological systems..$^{32,33}$

Recently other authors ${ }^{34}$ believe that a more accurate calculation of the real state of health or frailty of an individual is possible assessing the level of order or complexity of biodynamic signals through the measurement of entropy.

In this paper a signal analysis method based on Entropy (S), Lyapunov exponent (1), Median Absolute Deviation (MAD), Multiscale Entropy (MSE), is proposed to estimate the complexity of long-range temporal correlation heart rate (HR) time series for a healthy young man (30 yr old) and healthy elderly man ( 82 yr old).

Figure 2 shows the heart rate (HR) time series for a healthy young person (entropy value is 5.89) and Figure 3 shows the HR series for an healthy elderly person (with entropy value of 3.88). In this last case, the cardiac dynamics appear very different with respect to the dynamics of the young person, in fact the lower complexity of the signal is evident and therefore its low entropy value.

We further analyzed the two data sets (young and old) through the Lyapunov exponent $(\lambda)$, of the median absolute deviation (MAD) and MSE.

The data analysis of the two time series shows:

Elderly Subject: E: 3.88 MAD: 0.4438 MSE:1.47 1: $-0.0012 \mathrm{~T}_{1}:-$

Young Subject: E: 5.89 MAD: 3.3967 MSE:2.09 1: $0.00811 \mathrm{~T}_{1}: 123.26$

The young subject has a much higher MAD value than the elderly subject and this is justified by the fact that, as is also shown in Figure 2, data of the young person are much more grouped than the other data series.

In the elderly subject, the maximum exponent of lyapunov has a very small negative value $(1:-0.0012)$ that shows a regular cardiac dynamics. In contrast, in the young subject, the positive value of $\lambda$ shows the presence of a chaotic dynamic with a lyapunov time of $\mathrm{T}_{1}: 123.26(\mathrm{~s})$.

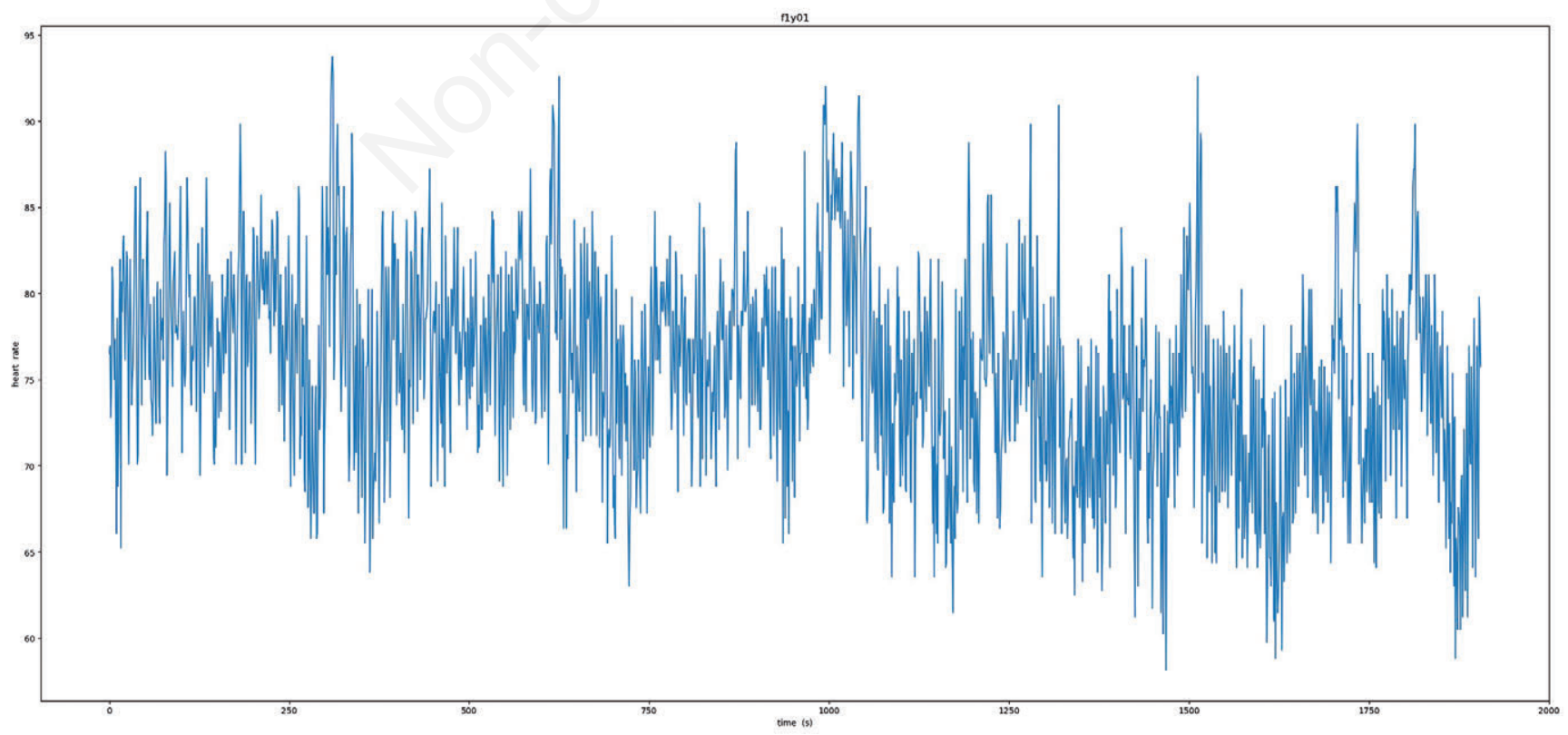

Figure 2. Heart rate time series for a healthy young man $(30 \mathrm{yr})$. 
To quantify, therefore, the nonlinear value of $E$ and $\lambda$ of a biological signal, is equivalent to measure the complexity of the dynamical system examined.

The higth valus of MSE in the time serie of the young subject, indicate the presence of self-similarity properties and therefore fractal properties.

Unlike the conventional idea of illness and old age in medicine a state of health is described by a state of normal irregularity (chaotic state) while the disease or old age would be characterized by an increase in regularity. Life, according to Eigen's studies $^{35}$ (Quasispecies theory) arises in the transition zone between order and chaos precisely where fractal structures are born.

We wish to emphasize that the agerelated loss of fractal organization in HR dynamics reflect the degradation of physiological regulatory systems. Furthermore, the entropy values set as variable within a Poisson distribution can predict the level of personal vulnerability. ${ }^{34}$ The poisson distribution, is considered a valid conceptual model to describe the evolution of the state of health of a particular organ or whole body.

\section{Conclusions}

In recent years, many research groups are trying to quantify the physiological signals of an individual, proposing new models to assess the complex dynamics of biologi- cal control systems. These new methods could improve overall understanding of the physiological system of the human organism, by adopting new models and experimental paradigms, such as those of fractality and entropy and studying, at the same time, the chaoticity of the biodynamic signal through Lyonunov exponent and the value of Multiscale Entropy, which have the ability to direct from an organ medicine to a modern systemic medicine.

The conceptual hypothesis that links aging, frailty, chaotic state or regularity and disease, suggests new methods for identifying possible future health conditions in relation to biodynamic signals described as changes in the values of entropy and Lyonunov exponent. In fact, considering the level of order or complexity of the anatomical apparatus by measuring the physical quantities, which are the entropy, the MSE and the $\lambda$, we could evaluate the health status or vice versa fragility of a biological system.

A relevant aspect is that such knowledge is early compared to those who can supply the traditional methods of clinical investigation.

\section{References}

1. Fried LP, Tangen CM, Walston J, et al. Frailty in older adults. J Gerontol Med Sci 2001;56A:M146-56.

2. Morley JE, Perry HM III, Miller DK.
Something about frailty. J Gerontol Med Sci 2002;57A:M698-704.

3. Woo J, Goggins W, Sham A, Ho SC. Public health significance of the frailty index. Disabil Rehabil 2006;28:515-21.

4. Palazzo S, Siciliano R, Turano S. Book: Neoplasie dell'anziano - cap. 4. Lecce: Ed. Milella; 2010.

5. Kulminski A, Yashin A, Arbeev K, et al. Cumulative index of health disorders as an indicator of aging-associatied processes in the elderly: results from analyses of the National Long Term Care Survey. Mech Ageing Dev 2007;128: 250-8.

6. Aktaruzzaman M, Migliorini M, Tenhunen $\mathrm{M}$, et al. The addition of entropy-based regularity parameters improves sleep stage classification based on heart rate variability. Med Biol Engine Comput 2015;53:415-25.

7. Bienertová-Vašku J, Zlámal F, Nečesánek I, et al. Calculating stress: from entropy to a thermodynamic concept of health and disease. PLoS One 2016;11:e0146667.

8. Goldberger AL, Amaral LAN, Glass L, et al. PhysioBank, PhysioToolkit, and PhysioNet: components of a new research resource for complex physiologic signals. Circulation 2000;101: e215-20.

9. Vidaurre C, Sander TH, Schlögl A. BioSig: the free and open source software library for biomedical signal processing. Comput Intell Neurosci 2011;2011:Article ID935364.

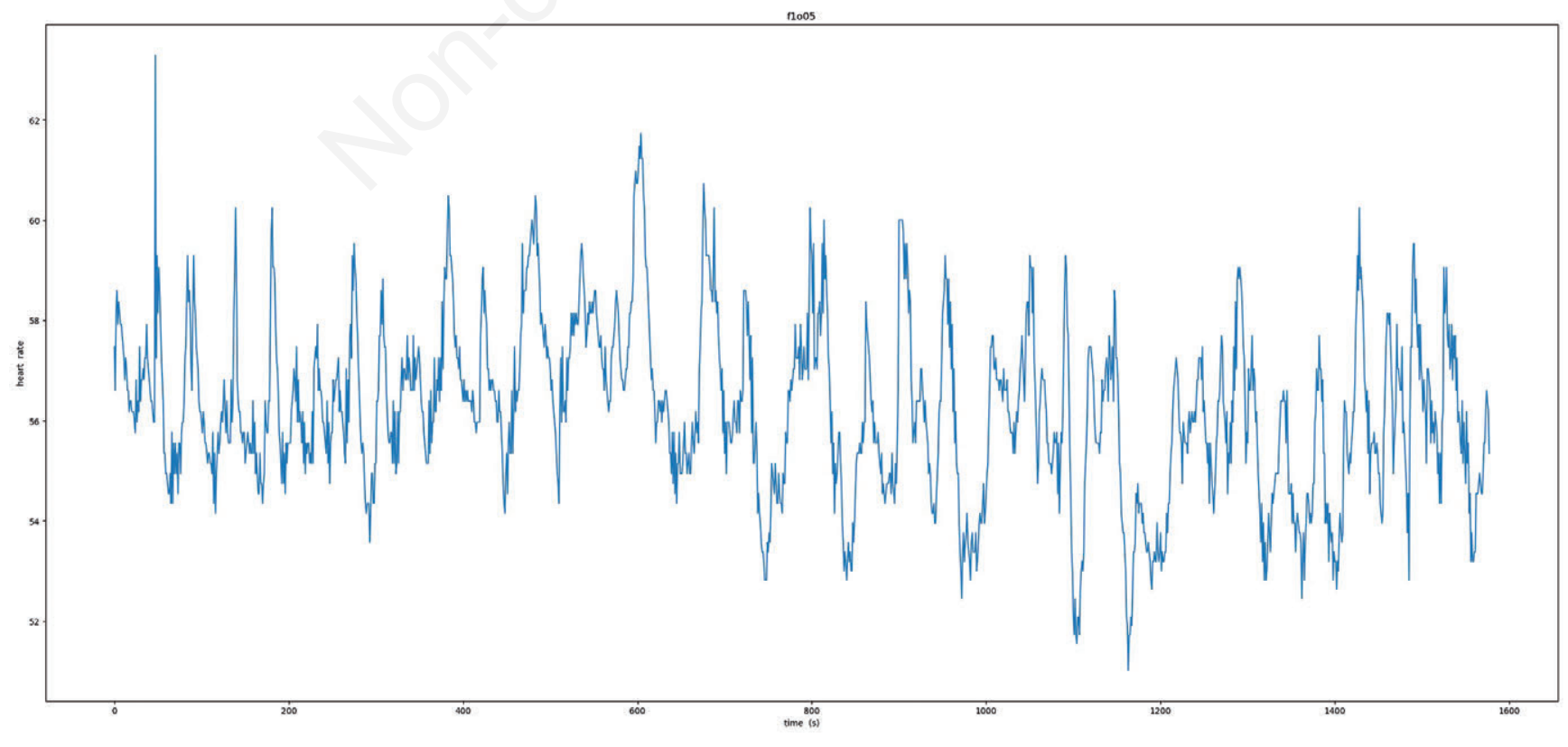

Figure 3. Heart rate time series for a healthy elderly man (82 yr). 
10. Tarvainen MP, Niskanen J-P, Lipponen JA, et al. Heart rate variability analysis software. Comput Method Program Biomed 2014;113:210-20.

11. Costa M, Goldberger AL, Peng C-K. Multiscale entropy analysis of biological signals. Phys Rev E 2005;71: 021906.

12. Costa M, Goldberger AL, Peng C-K. Multiscale entropy analysis of physiologic time series. Phys Rev Lett 2002; 89:062102.

13. Richman JS, Moorman JR. Physiological time-series analysis using approximate entropy and sample entropy. Am J Physiol Heart Circ Physiol 2000;278:H2039-49.

14. Pincus S.M. Approximate entropy as a measure of system complexity. Proc Natl Acad Sci U S A 1991;88:2297-301.

15. Lake DE, Richman JS, Griffin MP, Moorman JR. Sample entropy analysis of neonatal heart rate variability. Am J Physiol Regul Integr Comp Physiol 2002;283:R789-97.

16. Pincus SM. Assessing serial irregularity and its implications for health. Ann N Y Acad Sci 2002;954:245-67.

17. Lipsitz LA. Dynamics of stability: the physiologic basis of functional health and frailty. $\mathrm{J}$ Gerontol Biol Sci 2002;57A:B115-25.

18. Yates FE. Self-organizing systems. In: Boyd CAR, Noble R, eds. The Logic of Life - The Challenge of Integrative Physiology. New York: Oxford
University Press; 1993. pp 189-218.

19. Wilson DM III, Bohr VA, McKinnon PJ. DNA damage, DNA repair, ageing and age-related disease. Mech Ageing Dev 2008;129:349-52.

20. Schumacher B, Garinis GA, Hoeijmakers JH. Age to survive: DNA damage and aging. Trends Genet 2008;24:77-85.

21. Hortelano M, Reilly RB, Cervigòn R. Multiscale entropy analysis of heart rate regularity changes in older people with orthostatic intolerance. XIV Mediterranean Conference on Medical an Biological Engineering and Computing; 2016.

22. Fischer R, Akay M, Castiglioni P, Di Rienzo M. Multi-and monofractal indices of short-term heart rate variability. Med Biol Engine Comput 2003;41:543-9.

23. Cuesta D, Varela M, Miro P, et al. Predicting survival in critical patients by use of body temperature regularity measurement based on approximate entropy. Med Biol Engine Comput 2007;45:671-8.

24. Mandelbrot BB. The fractal geometry of nature. New York: Freeman; 1983.

25. Peng CK, Havlin S, Stanley HE, Goldberger AL. Quantification of scaling exponents and crossover phenomena in nonstationary heartbeat time series. Chaos 1995;5:82-7.

26. Duarte M, Sternad D. Complexity of human postural control in young and older adults during prolonged standing. Exp Brain Res 2008;191:265-76.

27. Abasolo D, Escudero J, Hornero R, et al. Approximate entropy and auto mutual information analysis of the electroencephalogram in Alzheimer's disease patients. Med Biol Engine Comput 2008;46:1019-28.

28. Li P, Liu CY, Sun X, et al. Assessing the complexity of short-term heartbeat interval series by distance distribution entropy. Med Biol Engine Comput 2015;53:77-87.

29. Anderson PW. More is different. Science 1972;177:393-6.

30. Yu MB. The entropy production of a nonequilibrium open system. J Phys 2015;6:128.

31. Zmeskal O, Dzik P, Vesely M. Entropy of fractal systems. Comput Math Appl 2013;66:135-46

32. Costa M, Goldberger AL, Peng C-K. Multiscale entropy analysis of complex physiological time series. Phys Rev Lett 2002;89:068102.

33. Lipsitz LA. Physiological complexity, aging, and the path to frailty. Sci Aging Knowl Environ 2004;16:16.

34. Siciliano R. Entropy as a method to investigate complex biological systems. An alternative view on the biological transition from healthy aging to frailty. Geriatric Care 2017;3:6755.

35. Eigen M, Schuster P. The hypercycle: a principle of natural self-organization. Berlin: Springer; 1979. 